\title{
Dos nuevos registros de margay (Leopardus wiedii) en Oaxaca, México, con datos sobre hábitos alimentarios
}

\author{
Claudia Cristina Cinta-Magallón ${ }^{1}$, Carlos Raúl Bonilla-Ruz ${ }^{2}$, Iván Alarcón-D. ${ }^{3}$ y Joaquín Arroyo-Cabrales ${ }^{3}$ \\ ${ }^{1}$ Investigador Independiente; Paseo de las Flores 340-4 Edif. Río Edén A, Col. Guadalupe Victoria, 38417, Puerto Vallarta, Jalisco, México; \\ jaguara71@gmail.com \\ ${ }^{2}$ Centro Interdisciplinario de Investigación para el Desarrollo Integral Regional unidad Oaxaca. Calle Hornos No. 1003, Indeco Xoxo, Xoxocotlán, \\ 711230 Oaxaca, México; cbonill@hotmail.com \\ ${ }^{3}$ Laboratorio de Arqueozoología, Instituto Nacional de Antropología e Historia, Moneda 16, Col. Centro, 06060, México, D.F.; \\ kakarotto4n@hotmail.com, arromatu@hotmail.com
}

Recibido 7-XI-2011 Corregido 02-I-2012 Aceptado 23-I-2012

\section{ABSTRACT}

Two new records of margay (Leopardus wiedii) in Oaxaca, Mexico, with data of food habits. Because of its nocturnal habits, the margay is a felid that is not commonly represented in scientific collections, although there are quite a few studies about their biology. In the State of Oaxaca, Mexico, the species was first documented in 1894 and until now there are only 13 records distributed in different geopolitical regions of the state, including the Tehuantepec Istmus, La Cañada, North and South Sierra. The recordings of this feline are only distributed on the windward side of the mountains of the Isthmus of Tehuantepec and in the northern and southern areas of the state, leaving large regions without information. This study documents the presence of this cryptic feline in the transition area between Sierra Norte and La Cañada and Mixe Sierra, of northern Oaxaca State, at two localities, one within a cloud forest and the other within an oak forest. The records are based on DNA analysis of scats. Taxonomic identification and analysis of skeletal remains recovered from the scats indicates that shrews are frequent prey in the dry season at San Andres Yaa and in the rainy season at San Juan Teponaxtla.

\section{KEY WORDS}

Margay, Leopardus wiedii, food habits, Oaxaca, small felids.

\section{RESUMEN}

El margay (Leopardus wiedii) es el felino más pequeño de México, habita en diversos tipos de vegetación, prefiere las selvas con cobertura vegetal muy densa, en donde abunden árboles grandes. Por sus hábitos nocturnos y su aparente rareza es una especie que se encuentra poco representada en las colecciones científicas y son pocos los estudios sobre su biología. En el estado de Oaxaca al momento se tienen solo 13 registros. Estos felinos sólo se distribuyen a barlovento de las elevaciones montañosas en el Istmo de Tehuantepec y del norte y sur del estado, dejando grandes espacios sin información dentro del territorio. El presente trabajo registra la presencia de este críptico felino en el área de transición entre la Sierra Norte y la Cañada y en la Sierra Mixe, al norte del estado. Los registros se obtuvieron a partir de la recolección de excrementos en campo y su identificación se realizó por medio de análisis de ADN. La musaraña forma parte importante de la dieta del tigrillo durante la temporada de secas en San Andres Yaa y durante la temporada de lluvias en San Juan Teponaxtla.

\section{PALABRAS CLAVE}

Margay, Leopardus wiedii, hábitos alimentarios, Oaxaca, felinos.
El margay o tigrillo (Leopardus wiedii) habita bosques tropicales, perennifolios, subcaducifolios, manglares y bosques mesófilo de montaña. Prefieren las selvas con cobertura vegetal muy densa, en donde abunden árboles grandes ya que duerme, descansa y caza principalmente en los árboles, aunque también lo hace en tierra. Se le encuentra desde el nivel del mar hasta los 1000msnm con escasos registro hasta los 3000msnm (Oliveira 1998, Aranda 2005, Wainwright 2007).

En México se distribuye en las llanuras costeras del Pacífico y Golfo de México y se unen ambas poblaciones en el centro del país, hacia el Istmo de Tehuantepec y la Península de Yucatán (Hall 1981, Aranda 2000, Aranda 2005). 
Los estudios sobre la dieta de los carnívoros proveen información sobre los pequeños mamíferos que evaden la detección del trampeo (Camardella et al. 2000). Respecto a la dieta del margay existe información limitada, ya que es muy difícil encontrar excrementos en el campo y no defeca en los caminos como otras especies de felinos (Aranda 2000, Caso comunicación personal 2010).

Se sabe que el margay se alimenta principalmente de especies animales de hábitos arbóreos y nocturnos pero también cazan especies terrestres. Su dieta se compone principalmente de pequeños roedores, aves, reptiles, frutos e insectos (Oliveira 1998, De la Rosa \& Nocke 2000). Se han hecho algunos estudios con base en la colecta de excremento o contenidos estomacales, presentándose gran variedad de alimentos en distintos países de América Latina.

En el presente trabajo se hace una recopilación de lo publicado sobre la distribución de la especie en el estado de Oaxaca, México, así como lo conocido sobre sus hábitos alimentarios y los datos que se conocen sobre parásitos en la especie. Se describe la presencia del margay en dos localidades de nuevo registro de la Sierra Norte de Oaxaca y con base en la colecta de excrementos y rastros; se describen los hábitos alimentarios de la especie en ambas localidades.

\section{Antecedentes}

\section{Registros de Leopardus wiedii}

En el estado de Oaxaca el primer registro de tigrillo o margay se presentó en 1894 (Goodwin 1969) y al momento se tienen sólo 13 registros de colecciones científicas, que aunque distribuidos en diversas regiones del estado, que abarcan el Istmo de Tehuantepec, la Cañada y la Sierra Norte y Sur de Oaxaca, sólo se distribuyen a barlovento de las elevaciones montañosas en el Istmo de Tehuantepec y del norte y sur del estado, dejando grandes espacios sin información dentro del territorio (Cuadro 1).

Otros registros basados en estudios realizados en el estado, incluyen el registro de Alfaro Espinosa (2006), los de Cinta Magallón (2007) quién reporta la presencia del tigrillo en 17 localidades de la Sierra Norte, basados en entrevistas y en la presencia de ejemplares disecados o en cautiverio y el de Pérez Irineo (2008), dentro de una selva mediana en la región de Tuxtepec (Cuadro 2).

\section{Hábitos alimentarios}

En Venezuela Mondolfi (1986) reportó la depredación del margay sobre mono capuchino (Cebus olivaceus), ardiIlas (Sciurus granatensis), rata de cañaveral (Zygodontomys brevicauda), ratón de abazones (Heteromys anomalus) y diversos anfibios. En Panamá se registra al tlacuache (Didelphis marsupialis) como parte de su alimentación (Goldman 1943). En Guatemala se encontró dentro del estómago de un margay a un ratón espinoso (Heteromys sp.; Oliveira 1994). En Brasil, en el estómago de un margay se encontraron restos de cuyos, Cavia fulgida y Galera spixii, plumas de tinamú (Tinamus solitarius) y piel y huesos de una rata de agua (Scapteromy sp.; Ximénez 1982). Mientras Azevedo (1996) reporta pollos y anfibios. Asimismo Wang (2002), para el sur de Brasil reporta mamíferos de talla pequeña y mediana, terrestres y nocturnos, aves y serpientes en la dieta del tigrillo. Bianchi et al. (2011), en la selva lluviosa Atlántica de Brasil reporta como dieta principal del margay al conejo (Sylvilagus brasiliensis) y el armadillo (Dasypus sp.) en los remanentes de bosque de la misma área. Rocha Mendes et al. (2010), concluyeron en su estudio sobre la dieta de los carnívoros que el tigrillo se alimenta primordialmente de roedores, marsupiales y aves de manera incidental, también se conoce la ingesta de murciélagos (Rocha Mendes \& Bianconi 2009).

Ya más al norte Beebe (1925), reporta en la Guyana Francesa un perezoso de tres dedos (Bradypus tridactylus), un mico maicero (Cebus apella), un puercoespín (Coendou prehensilis) y un momoto coroniazul (Momotus momota) en la dieta del tigrillo. En Belice, Konecny (1989) encontró una rata trepadora (Ototylomys phyllotis) como la más común en la dieta del margay, así como otras especies incluyendo el tlacuache cuatro ojos (Philander opossum), pequeñas aves y ardillas (Sciurus deppei), así como también insectos y frutos, aunque no son parte importante de su dieta. En cautiverio se les ha visto alimentarse con higos y hasta con lechuga y en alguna ocasión se le observó depredando un tamarino de manos negras (Saguinus midas niger), una rata (Rattus norvegicus), lagartijas (Tropidurus sp.) y pequeñas aves paserinas (Petersen 1971, Oliveira 1998).

En México referente a la alimentación del margay, Álvarez del Toro (1977), menciona que en Chiapas se alimentan de ratones de campo, conejos, pacas, agutis jóvenes, aves y ocasionalmente crías de temazate (Mazama temama).

Aunque la mayoría de los alimentos que consumen son especies pequeñas, se piensa que el reporte de especies medianas se debe a que las consumen como carroña, ya que la morfología de su cabeza y sus dientes no tienen la fuerza para atacar y derribar presas de estas características (Oliveira 1998, Wang 2002).

A partir del análisis de excrementos se han hallado evidencias de endoparásitos. Los parásitos registrados para tigrillo son: Toxoplasma gondii, Dirofilaria striata, (Bowman et al. 2002), Toxocara sp. y Aelurostrongylus abstrusus (Carbajal Villareal 2005). 


\section{CUADRO 1}

Registros de margay en colecciones científicas para el estado de Oaxaca

\begin{tabular}{|c|c|}
\hline Localidad & Fuente \\
\hline \multicolumn{2}{|l|}{ Leopardus wiedii } \\
\hline Ixtlán de Juárez ca. 55 Km N & Carnegie Museum \\
\hline $\begin{array}{l}\text { Cafetal Carlota, Cueva } \\
\text { Carretera Huautla-San } \\
\text { Bartolomé Ayautla }\end{array}$ & CNM \\
\hline $\begin{array}{l}\text { La Soledad, San Bartolomé } \\
\text { Ayautla, Teotitlán }\end{array}$ & CNM \\
\hline Cerro Baúl, Rancho San Vicente & LACM \\
\hline San Gabriel Mixtepec, $7 \mathrm{Km} \mathrm{N}$ & LACM \\
\hline Concepción Pápalo & IBUNAM (Filmoteca) \\
\hline Santiago Nacaltepec & IBUNAM (Filmoteca) \\
\hline \multicolumn{2}{|l|}{ Leopardus wiedii oaxacensis } \\
\hline Laguna Guelaguichi & CNM \\
\hline $\begin{array}{l}\text { Cerro San Felipe, } 10 \mathrm{mi} \mathrm{N} \text { de } \\
\text { Oaxaca }\end{array}$ & USNM \\
\hline La Gloria Juchitán & AMNH \\
\hline La Gloria Juchitán & AMNH \\
\hline Santa Lucía Tehuantepec & AMNH \\
\hline \multicolumn{2}{|l|}{ Leopardus wiedii yucatanica } \\
\hline Ocotal Juchitan & AMNH \\
\hline
\end{tabular}

Las abreviaturas corresponden a: LACM: Museo de Historia Natural del Condado de Los Ángeles. AMNH: Museo Americano de Historia Natural; USNM: Museo Nacional de Historia Natural de los Estados Unidos: CNM: Colección Nacional de Mamíferos, IBUNAM: Instituto de Biología, Universidad Nacional Autónoma de México.

\section{METODOLOGÍA}

Las localidades donde se encontraron evidencias de margay (Leopardus wiedii) como excrementos y un comedero se describen a continuación:

\section{San Andrés Yaa}

Se encuentra en el municipio del mismo nombre, en las coordenadas $17^{\circ} 17^{\prime} 30^{\prime \prime} \mathrm{N}, 96^{\circ} 00^{\prime} 11^{\prime \prime} \mathrm{W}$; pertenece al distrito de Villa Alta, a una altura de $1560 \mathrm{msnm}$, los tipos de vegetación son bosque mesófilo de montaña, bosque de pino y bosque de encino con un clima templado húmedo (García 1999, SEMARNAT 2000).

\section{San Juan Teponaxtla}

Limita con la Cañada, pertenece al Municipio de San Juan Tepeuxila, en las coordenadas $17^{\circ} 43^{\prime} 02^{\prime \prime} \mathrm{N}$,
CUADRO 2

Registros de margay colectados en la Sierra Norte de Oaxaca

\begin{tabular}{|c|c|}
\hline Localidad & Autor \\
\hline Santiago Comaltepec & Alfaro Espinoza 2006 \\
\hline $\begin{array}{l}\text { ^Huautla de Jiménez, Huautla de } \\
\text { Jiménez }\end{array}$ & Cinta Magallón 2007 \\
\hline $\begin{array}{l}\text { ^San Bernardino, Teotitlán de Flores } \\
\text { Magón }\end{array}$ & Cinta Magallón 2007 \\
\hline $\begin{array}{l}\text { ^San Juan Coyula, San Juan Bautista } \\
\text { Cuicatlán }\end{array}$ & Cinta Magallón 2007 \\
\hline $\begin{array}{l}\text { ^San Gabriel Etla, San Juan Bautista } \\
\text { Guelache }\end{array}$ & Cinta Magallón 2007 \\
\hline $\begin{array}{l}\text { ^Arroyo Guacamaya, Teocuicuilco de } \\
\text { Marcos Pérez }\end{array}$ & Cinta Magallón 2007 \\
\hline ^Santa Catarina Ixtepejí & Cinta Magallón 2007 \\
\hline$\wedge$ Santiago Laxopa & Cinta Magallón 2007 \\
\hline ^San Pedro Cajonos & Cinta Magallón 2007 \\
\hline$\wedge$ Santiago Comaltepec & Cinta Magallón 2007 \\
\hline ^La Esperanza, Santiago Comaltepec & Cinta Magallón 2007 \\
\hline $\begin{array}{l}\text { ^San Martín Soyolapan, Santiago } \\
\text { Comaltepec }\end{array}$ & Cinta Magallón 2007 \\
\hline $\begin{array}{l}\text { ^San Gaspar Yagalaxí , Ixtlán de } \\
\text { Juárez }\end{array}$ & Cinta Magallón 2007 \\
\hline *San Pedro y San Pablo Ayutla* & Cinta Magallón 2007 \\
\hline $\begin{array}{l}\text { ^Rancho Tejas, Santa María } \\
\text { Tlahuitoltepec }\end{array}$ & Cinta Magallón 2007 \\
\hline $\begin{array}{l}\wedge \text { Tierra Blanca, Tamazulapan del } \\
\text { Espiritú Santo }\end{array}$ & Cinta Magallón 2007 \\
\hline +Totontepec Villa de Morelos & Cinta Magallón 2007 \\
\hline$\wedge$ Chayotepec , Santa Ma. Guienagati & Cinta Magallón 2007 \\
\hline${ }^{\circ}$ Cerro Tepezcuintle, Distrito de & Pérez Irineo 2008 \\
\hline
\end{tabular}

Tuxtepec

${ }^{\circ}$ Rastro; $\wedge$ Entrevistas; ${ }^{*}$ Animales disecados; + animal en cautiverio.

$96^{\circ} 43^{\prime} 15^{\prime \prime} \mathrm{W}$, dentro del distrito de Cuicatlán, a una altura de $1560 \mathrm{msnm}$, los tipos de vegetación son bosque mesófilo de montaña, bosque de pino y bosque de encino con un clima semicálido (García 1999, SEMARNAT 2001).

\section{Recolección y procesamiento de muestras}

Se recolectaron excrementos y otros rastros en el área de estudio al final de la temporada de secas para San Andrés Yaa (mayo) y a principios de la temporada de lluvias para San Juan Teponaxtla (junio). Todos los excrementos fueron fotografiados, georeferenciados y secados a 
temperatura ambiente. Una porción de aproximadamente $1 \mathrm{gr}$ de cada excremento, fue enviada al programa del Centro de Genética de la Conservación y Genética Global de Felinos, en el Museo Americano de Historia Natural (Center for Conservation Genetics and Global Felid Genetics), donde mediante análisis de ADN se determinó la especie a la que dichos excrementos pertenecen. Otra pequeña porción, aproximadamente 1 o 2 gramos, de cada excremento en el momento de encontrarla, fue conservada en alcohol al $70 \%$, para realizar un análisis coproparasitoscópico por el método cuantitativo de flotación en saturación de cloruro de sodio $(\mathrm{NaCl})$ o método de Willis (Segovia de Romero \& Ozuna 2000). El análisis se llevó a cabo en el Laboratorio de Parasitología de la Facultad de Medicina Veterinaria y Zootecnia en la Facultad de Estudios Superiores-Cuautitlán, Universidad Nacional Autónoma de México. Después de éste análisis, el material utilizado de cada excremento, fue secado a temperatura ambiente y reincorporado a sus excrementos originales, previamente secados para la realización del análisis de contenido.

Posteriormente se procedió a la separación del material óseo y partículas reconocibles de material animal, que se entregó para su identificación al Laboratorio de Arqueozoología "M en C. Ticul Álvarez Solorzano", Subdirección de Laboratorios y Apoyo Académico, Instituto Nacional de Antropología e Historia. Los restos animales encontrados se determinaron al nivel taxonómico más fino posible.

Otra fuente de información sobre los hábitos alimentarios de $L$. wiedii a través del trabajo de campo, proviene de tomar en cuenta las características de los sitios que utilizaron los tigrillos para comer y de los rastros y huellas diferentes a excrementos que pudieran encontrarse en éstos, como plumas, huesos y otro tipo de resto animal con signos de haber sido consumidos por el margay.

El material así colectado y relacionado con los excrementos, fue identificado con material de referencia de colecciones regionales y nacionales, como la colección regional de aves del Centro Interdisciplinario de Investigación para el Desarrollo Integral Regional (CIIDIR) Oaxaca (Oax-Ave).

\section{RESULTADOS}

Se colectaron 14 excrementos secos en las localidades de San Andrés Yaa (10 excrementos) y en San Juan Teponaxtla (4 excrementos), de estos 11 pertenecieron a puma (Puma concolor) y solo tres pertenecieron a margay (Leopardus wiedii), de acuerdo a los resultados del análisis de ADN. De esta forma, se adicionaron las dos localidades para el tigrillo en el estado de Oaxaca descritas en la sección de metodología.
Un excremento y los datos del comedero fueron colectados en terrenos de San Andrés Yaa, dentro de una vegetación de bosque de encino en la que predominaba un clima templado húmedo; el excremento fue encontrado a una altura de $2125 \mathrm{msnm}$ en las coordenadas $17^{\circ} 15^{\prime} 46.6^{\prime \prime} \mathrm{N}$ y $96^{\circ} 06^{\prime} 17^{\prime \prime}$ W. En el nudo de un tronco de árbol aproximadamente a dos metros del suelo, se encontraron gran cantidad de plumas de color azul y huellas asociadas en el suelo, las plumas se colectaron y con ayuda de las fotografías tomadas en el lugar y los ejemplares consultados en la colección de aves del CIIDIR Oaxaca, se determinó que pertenecían a una chara azuleja o urraca azuleja (Aphelocoma caerulescens). El comedero se encontraba a una altura de $2100 \mathrm{msnm}$ en las coordenadas $17^{\circ} 16^{\prime} 15.1^{\prime \prime} \mathrm{N}$ y $96^{\circ} 06^{\prime} 27^{\prime \prime} \mathrm{W}$, ambos registros fueron recabados el 27 de mayo de 2006.

Dos excrementos fueron recolectados en terrenos de San Juan Teponaxtla; en uno de los sitios predominaba un clima templado húmedo, a una altura de $2450 \mathrm{msnm}$ en las coordenadas $17^{\circ} 45^{\prime} 18.8^{\prime \prime} \mathrm{N}$ y $96^{\circ} 43^{\prime} 00.12^{\prime \prime} \mathrm{W}$. El otro sitio se encontró en un bosque mesófilo de montaña con clima semifrío subhúmedo, a una altura de 2436 msnm, en las coordenadas $17^{\circ} 45^{\prime} 06.6^{\prime \prime} \mathrm{N}$ y $96^{\circ} 43^{\prime} 00.14^{\prime \prime} \mathrm{W}$; ambos registros se tomaron el 22 de junio de 2006.

\section{Análisis de contenido de excremento}

Se identificaron 87 fragmentos óseos o dientes, los cuales representan al menos 10 especies de vertebrados pertenecientes a tres clases: Reptilia, Aves y Mammalia (Cuadro 3).

Dentro de Reptilia, dos familias están representadas, Anguidae con tres restos, dos de ellos son fragmentos de las regiones frontal y occipital del cráneo (área del "ojo pineal"), probablemente perteneciente a una lagartija del género Mesaspis, tentativamente identificada como M. cf. juarezi mientras que para la familia Phyronosomatidae, existen dos ejemplares de lagartija asignados al género Sceloporus, una mandíbula corresponde más cercanamente a S. cf. variabilis.

Para la clase de las aves varios fragmentos de huesos se separaron, pero sólo fue posible identificar un fragmento distal de tibiotarso como perteneciente a una chachalaca Ortalis cf. vetula.

La clase más diversa fueron los mamíferos (Mammalia) con cinco especies pertenecientes a dos órdenes: Soricomorpha y Rodentia. Dos especies de musaraña pertenecientes al mismo género se identifican de manera tentativa debido a la ausencia de material biológico para comparar directamente, Cryptotis cf. goldmani y C. cf. mexicana, ambas especies que pudieran hallarse en 
la región de donde proceden las muestras (Woodman \& Timm 1993, 1999).

Dentro de los roedores, dos familias están representadas: Sciuridae y Cricetidae. La ardilla Sciurus auerogaster está representada por dos fragmentos óseos, uno de fíbula y otro de húmero. De los ratones, se tienen ejemplares pertenecientes a dos especies del ratón cuatroalbo ( $\mathrm{Pe}$ romyscus), P. cf. maniculatus y P. mexicanus (Cuadro 4).

En el análisis coproparasitoscópico por el método de Willis no se halló algún tipo de huevo o parásito, no se hicieron otro tipo de análisis porque los excrementos no se encontraban frescos al momento de la colecta.

\section{DISCUSIÓN}

El área de distribución del margay ocupa la mayor parte del estado; sin embargo, son pocos los registros que se tienen de él; de esta forma, las dos nuevas localidades con presencia de margay para el estado de Oaxaca enriquecen el conocimiento sobre su distribución en la entidad. Se ha

\section{CUADRO 3}

Especies identificadas procedentes de excrementos de L. wiedii de la región de San Juan Teponaxtla (Muestras 1 y 2) y San Andrés Yaa, Oaxaca. Bajo cada sitio de colecta se reportan los individuos representados por los restos óseos.

\begin{tabular}{|c|c|c|c|}
\hline Especie & Elementos óseos identificados & $\mathbf{N}$ & NMI \\
\hline \multicolumn{4}{|c|}{ San Juan Teponaxtla ( muestra 1) } \\
\hline Mesaspis juarezi & Un fragmento del cráneo, osteodermos y un fragmento de cráneo. & 3 & 1 \\
\hline Sceloporus sp. & Escamas & & 1 \\
\hline Ortalis cf. vetula & Un fragmento distal del tibiotarso & 1 & 1 \\
\hline Criptotis cf. goldmani & 3 fragmentos de mandíbula, 4 húmeros izquierdos y 2 derechos & 9 & 4 \\
\hline Cryptotis cf. mexicana & $\begin{array}{l}4 \text { fragmentos de mandíbula derecha y } 2 \text { de mandíbula izquierda, } 2 \\
\text { fragmentos proximales del maxilar, } 3 \text { del maxilar derecho y } 2 \text { del izquierdo }\end{array}$ & 13 & 5 \\
\hline Sciurus aureogaster & Un fragmentos proximal del húmero y un fragmento proximal de fíbula & 2 & 1 \\
\hline Peromyscus cf. maniculatus & $\begin{array}{l}3 \text { fragmentos de mandíbula izquierda y uno de mandíbula derecha, } 3 \\
\text { molares inferiores m } 1 \text { más } 14 \text { molares aislados. }\end{array}$ & 21 & 3 \\
\hline Peromyscus mexicanus & $\begin{array}{l}\text { Un fragmento del maxilar, uno de la mandíbula, } 3 \text { molares inferiores m1, } \\
\text { más } 9 \text { molares }\end{array}$ & 14 & 3 \\
\hline Subtotal & & 63 & 20 \\
\hline \multicolumn{4}{|c|}{ San Juan Teponaxtla (Muestra 2) } \\
\hline Mesaspis cf. juarezi & Un fragmento de cráneo ojo pineal & 1 & 1 \\
\hline Sceloporus cf. variabilis & Un fragmento de mandíbula y uno de pelvis & 2 & 1 \\
\hline Cryptotis cf. goldmani & 2 húmeros derechos & 2 & 2 \\
\hline Cryptotis cf. mexicana & $\begin{array}{l}4 \text { fragmentos del maxilar, } 6 \text { fragmentos de mandíbulas } 3 \text { izquierdas y } \\
3 \text { derechas y } 3 \text { húmeros }\end{array}$ & 13 & 4 \\
\hline Peromyscus cf. maniculatus & Primer molar inferior m1 y 1 fragmento de la mandíbula izquierda & 2 & 1 \\
\hline Subtotal & & 20 & 9 \\
\hline \multicolumn{4}{|l|}{ San Andrés Yaa } \\
\hline Cryptotis cf. goldmani & Un fragmento de mandíbula & 1 & 1 \\
\hline Cryptotis cf. mexicana & Un par de húmeros, izquierdo y derecho, un fragmento de maxilar & 3 & 1 \\
\hline Subtotal & & 4 & 2 \\
\hline Total & & 87 & 31 \\
\hline
\end{tabular}




\section{CUADRO 4}

Identificación del material orgánico animal encontrado en excrementos de $L$. wiedii en el norte de Oaxaca, México.

\begin{tabular}{|c|c|c|}
\hline Clase / Orden & Familia & Género y especie \\
\hline \multicolumn{3}{|l|}{ Reptilia } \\
\hline Squamata & Phrynosomatidae & Sceloporus sp. \\
\hline Squamata & Phrynosomatidae & Sceloporus cf. variabilis \\
\hline Squamata & Anguidae & Mesaspis cf. juarezi \\
\hline \multicolumn{3}{|l|}{ Aves } \\
\hline Galliformes & Cracidae & Ortalis cf. vetula \\
\hline Passeriformes & Corvidae & Aphelocoma caerulesens* \\
\hline \multicolumn{3}{|l|}{ Mammalia } \\
\hline Soricomorpha & Soricidae & Cryptotis cf. mexicana \\
\hline Soricomorpha & Soricidae & Cryptotis cf. goldmani \\
\hline Rodentia & Sciuridae & Sciurus aureogaster \\
\hline Rodentia & Cricetidae & Peromyscus cf. maniculatus \\
\hline Rodentia & Cricetidae & Peromyscus mexicanus \\
\hline
\end{tabular}

*Restos encontrados en el comedero.

registrado que el tigrillo rara vez se encuentra por arriba de los 1200msnm (Eisenberg 1990), siendo excepcionales los registros a entre 1200 y 3000msnm (Aranda 2005); sin embargo, los registros aquí presentados se encontraron a un promedio de $2277 \mathrm{msnm}$.

Se dice que estos animales no defecan en caminos ocupados por el hombre (Aranda 2000) y que tienen la tendencia de cubrir los excrementos con hojas y tierra, depositándolos en lugares conspicuos (Emmons \& Feer 1997); sin embargo, dos de los excrementos fueron encontrados en caminos utilizados por el hombre y los tres se encontraban sin cubrir.

Todas las especies identificadas en los excrementos, corresponden con animales que actualmente se encuentran en la región de donde éstas proceden. Es interesante señalar que los restos más abundantes en los excrementos fueron de musarañas $y$, en segundo término de ratones. La biología de las musarañas es poco conocida y aquí se adiciona información relevante acerca de sus depredadores. Quizás estas localidades sean adecuadas para un estudio a largo plazo de estos pequeños mamíferos.

En la mayor parte de la literatura se reporta que el tigrillo tiene una dieta compuesta principalmente por mamíferos de hábitos arbóreos (Guggisberg 1975, Konecny 1989, Oliveira 1994) y aves (Leyhausen 1990), lo que se relaciona con su morfología adaptada a habitar en los árboles (Oliveira 1994); sin embargo según publicaciones más recientes, estos felinos cazan mamíferos de hábitos terrestres, así como en menor grado aves (Moldolfi 1986, Ximenez 1982, Azevedo 1996, Facure \& Giaretta 1996, Wang 2002, Rocha Mendes et al. 2010, Bianchi et al. 2011). Los resultados del presente estudio corroboran tal información, confirmando que es un felino de hábitos generalistas (Wang 2002).

Hasta el momento, en los estudios realizados en otros países, no se ha reportado en la lista de animales consumidos por el margay a la musaraña. En este estudio se registran dos especies de musaraña dentro de sus excrementos, las cuales aparecen en ambas localidades, siendo estos animales aparentemente parte importante de su dieta, ya que de acuerdo a los datos de frecuencia de ocurrencia, pueden representar desde el $45 \%$ en el caso de la muestra 1 de San Juan Teponaxtla hasta un $100 \%$ en la muestra de San Andrés Yaa.

La ardilla es un mamífero continuamente mencionado como parte de la dieta del margay (Konecny 1989, Mondolfi 1996), confirmándose en el presente estudio.

Existen pocos estudios sobre los parásitos que afectan al tigrillo, algunos de los parásitos reportados no especifican la procedencia de los ejemplares infectados, el 
estudio realizado por Carbajal Villareal (2005) si menciona que los datos obtenidos fueron de animales en vida libre; en el presente trabajo las muestras fueron obtenidas de animales silvestres, pero los resultados de los estudios coproparasitoscópicos, no fueron concluyentes; se podría decir que aparentemente son animales sanos, sin embargo, dada la poca cantidad de muestras, no puede asegurarse que esta sea una condición general de la población de esta especie de felino en la región.

Trabajar con animales tan crípticos como lo es el tigrillo tiene un alto grado de dificultad, sería recomendable que el tamaño de muestra fuera mayor, sin embargo por el grado de dificultad para encontrar rastros de este felino la información obtenida en este trabajo es de gran valor, siendo el primero en su tipo en México y da la pauta para continuar con este tipo de estudios para conocer más sobre la biología de esta especie.

Se reportan dos nuevas localidades con presencia de tigrillo para el Estado de Oaxaca, en una altitud mayor a 2277msnm.

Los excrementos fueron encontrados en caminos utilizados por el hombre y sin cubrir. Información reciente argumenta que estos felinos no solo cazan animales de hábitos arbóreos y aves, sino que también cazan mamíferos de hábitos terrestres, esta información se ve corroborada en el presente estudio. Se confirma al tigrillo como un animal de hábitos generalistas.

La musaraña forma parte importante de la dieta del tigrillo durante la temporada de secas en San Andres Yaa y durante la temporada de lluvias en San Juan Teponaxtla. A pesar de que la ardilla es una presa común en todos los estudios previos sobre la dieta del margay, en este caso de acuerdo al análisis de excrementos, aunque está presente, no es una presa frecuente. Se marca la pauta para continuar con estudios parasitoscópicos para conocer los parásitos que afectan a esta especie.

\section{AGRADECIMIENTOS}

A John Polisar de Wildlife Conservation Society por su interés en el presente trabajo, mismo que hecemos extensivo a Salisa Rabinowitz del Centro para la Conservación Genética y Global de los felinos A.M.N.H. por realizar los análisis de ADN. A Pablo Martínez Lavat del laboratorio de Parasitología de la Fes-Cuautitlán UNAM, por su paciencia y asesoría en la búsqueda de parásitos en los excrementos.

A Emma Cisneros Palacios por las facilidades prestadas para consultar la Colección de Aves Ciidir Oaxaca IPN y compartir su conocimiento en la identificación de las plumas encontradas en un comedero de margay. A Cesar
Vasconcelos, habitante de Totontepec Villa de Morelos y a Eliseo Pérez habitante de San Juan Teponaxtla por ser guías dentro de sus terrenos y enriquecernos con el conocimiento que tienen de sus comunidades. Al Instituto Politécnico Nacional CIIDIR Oaxaca y al CONACyT por las facilidades otorgadas para llevar a cabo los estudios de postgrado y los apoyos económicos recibidos a través de sus becas.

\section{REFERENCIAS}

Alfaro Espinosa, A.M. 2006. Patrones de diversidad de mamíferos terrestres del municipio de Santiago Comaltepec, Oaxaca, México. Tesis para obtener el grado de Maestría en Ciencias en Conservación y Aprovechamiento de Recurso Naturales. Instituto Politécnico Nacional. Centro Interdisciplinario de Investigación para el Desarrollo Integral Regional. Oaxaca, México.

Álvarez del Toro, M. 1977. Los Mamíferos de Chiapas. México, Universidad Autónoma de Chiapas. Chiapas, México.

Aranda, M. 2000. Huellas y otros rastros de los mamíferos grandes y medianos de México. Instituto de Ecología A.C. y Comisión Nacional para el Conocimiento y uso de la Biodiversidad (CONABIO). Veracruz, México.

Aranda, M. 2005. Leopardus wiedii (Schinz, 1821) Tigrillo. Pp. 361362. In Ceballos \& Oliva (eds). Los Mamíferos Silvestres de México. Comisión Nacional pare el Conocimiento y Uso de la Biodiversidad y Fondo de Cultura Económica. México.

Azevedo, F.C.C. 1996. Notes on the behavior of the margay Felis wiedii (Schinz 1821) Carnivora, Felidae, in the Brazilian Atlantic Forest. Mammalia 60:325-328.

Beebe, W. 1925. Studies of a Tropical Jungle, one quarter of a square mile of jungle at Kartabo, Guiana Britanica. Zoologica 6: 1-193

Bianchi, R., A.F. Rosa., A. Gatti. \& S.L. Mendes. 2011. Diet of margay, Leopardus wiedii, and jaguarundi, Puma yagouaroundi, Carnivora, Felidae in Atlantic Rainforest, Brazil. Zoologia 28: 127-132.

Bowman, D.D., C.M. Hendrix, D.S. \& Lindsay, S.C. Barr. 2002. Feline Clinical Parasitology. lowa State University, lowa, EEUU.

Camardella A.R., M.F. Abrew \& E. Wang. 2000. Marsupials found in felid scats in southeastern Brazil, and a range extension of Monodelphis theresa. Mammalia 64: 379-382.

Carbajal Villareal S. 2005. Ámbito hogareño y patrón de actividad del Margay Leopardus wiedii (Schinz, 1821) en la Reserva de la Biosfera "El Cielo"Tamaulipas, México. Tesis para obtener el grado de Maestría en Ciencias en Biología. Instituto Tecnológico de Ciudad Victoria, División de estudios de Posgrado en Investigación, Tamaulipas, México.

Cinta Magallón C.C. 2007. Construcción y validación de un modelo de nicho ecológico para jaguar (Panthera onca) y puma (Puma concolor) en la Sierra Madre de Oaxaca, México. 
Tesis para obtener el grado de Maestría en Ciencias en Conservación y Aprovechamiento de Recurso Naturales. Instituto Politécnico Nacional. Centro Interdisciplinario de Investigación para el Desarrollo Integral Regional, Oaxaca, México.

De la Rosa C.L \& C.C. Nocke. 2000. A guide to the Carnivores of Central America. University of Texas, texas, EEUU.

Eisenberg, J.F. 1990. Mammals of the neotropics. Vol. 1:The Northern Neotropics. University of Chicago, Chicago, EEUU.

Emmons L.H. \& F. Feer. 1997. Neotropical Rain Forest Mammals a field guide. University of Chicago, Chicago, EEUU.

Facure, K.G. \& A.A. Giaretta. 1996. Food habits of carnivores in a costal Atlantic Forest of southeastern Brazil. Mammalia 603:499-502.

García A. 1999. Distritos, municipios y habitantes de Oaxaca. Ángel García García y Colaboradores, Oaxaca. México.

Goldman E.A. 1943. The races of ocelot and margay in Middle America. Journal of Mammalogy. 24: 372-385.

Goodwin G.G. 1969. Mammals from the state of Oaxaca, Mexico, in the American Museum of Natural History; Bulletin of the American Museum of Natural History. 141: 244-246.

Guggisberg C.A.W. 1975. Wild cats of the world. Taplinger, Nueva York, Nueva York, EEUU.

Hall R.E. 1981. The mammals of North America. Weley, Nueva York, Nueva York, EEUU.

Konecny M.J.1989. Movement patterns and food habits of four sympatric carnivore species in Belize, Central America. Advances in Neotropical Mammalogy. 243-264.

Leyhausen P. 1990. Cats. p: 576-632. In Grzimek's encyclopedia mammals. Van Nostrand Reinhold, Nueva York, Nueva York, EEUU.

Mondolfi E. 1986. Notes on the biology and status of the small wild cats in Venezuela. p. 125-146. In Cats of the world; biology, conservation and management, S.D. Miller, D.D. Everett (eds). National Wildlife Federation, Washington, Columbia, EEUU.

Oliveira T.G. 1994. Neotropical cats: ecology and conservation. Edufma, Sao Luís, Brasil.

Oliveira T.G. 1998. Leopardus wiedii. Mammalian species 579: 1-6.
Pérez Irineo G. 2008. Diversidad de mamíferos carnívoros terrestres en una selva mediana en el Distrito de Tuxtepec, Oaxaca. Tesis para obtener el grado de Maestría en Ciencias en Conservación y Aprovechamiento de Recurso Naturales. Instituto Politécnico Nacional. Centro Interdisciplinario de Investigación para el Desarrollo Integral Regional, Oaxaca, México.

Petersen M.K., 1971. Lettuce prevents stomach irritation in margay. Long Island Ocelot Club Newsletter 156:13

Rocha-Mendes F., S. Bos-Mikich., J. Quadros \& W.A. Pedro. 2010. Feeding ecology of carnivores Mammalia, (Carnivora) in Atlantic Forest remnants, Southern Brazil. Biota Neotropical. 10:4

Rocha Mendes F \& G.V. Bianconi. 2009. Opportunistic predatory behavior of margay, Leopardus wiedii (Schinz, 1821) in Brazil. Mammalia 73:151-152.

Segovia de Romero T. \& R. Ozuna Wood. 2000. Aspectos clínicos, terapéuticos y zoonóticos en las infestaciones gastrointestinales. Revista de Ciencia y Tecnología Dirección de Investigaciones-UNA. 1:97-103.

SEMARNAT/ Instituto de Geografía UNAM. 2000. Inventario Nacional Forestal. México.

Wainwright M. 2007. The mammals of Costa Rica. A natural history and field guide. A Zona Tropical publication. Costa Rica.

Wang E. 2002. Diets of Ocelots (Leopardus pardalis), Margays (L. wiedii), and Oncillas (L. tigrinus) in the Atlantic rainforest in southeast Brazil. Studies on Neotropical Fauna and Environment 37: 207-212

Woodman, N. \& R.M. Timm. 1993. Intraspecific and interspecific variation in the Cryptotis nigrescens species complex of small-eared shrews Insectivora: Soricidae, with the description of a new species from Colombia. Fieldiana: Zoology, new series, 74: iii+30.

Woodman, N. \& R.M. Timm. 1999. Geographic variation and evolutionary relationships among broad-clawed shrews of the Cryptotis goldmani-group Mammalia: Insectivora: Soricidae). Fieldiana: Zoology, new series, 91: 1-35.

Ximénez, A. 1982. Notas sobre felinos neotropicales, VIII. Observaciones del contenido estomacal y el comportamiento alimentario de diversas especies de felinos. Revista Nordestina de Biología. 5:89-91 